\title{
Unmöglichkeit guter Gitterpunktformeln
}

\author{
von
}

\author{
Klaus Langmann (Münster)
}

Bekannt ist, dass bei Gitterpunktproblemen wie z.B. beim Viertelkreis

$$
G(t):=\left\{(x, y) \in \mathbb{N}_{0}^{2} ; x^{2}+y^{2} \leq t\right\}
$$

Formeln wie etwa nachstehende Beziehung

$$
|G(t)|=\varphi(t)+\mathcal{O}\left(t^{1 / 4}\right) \quad \text { für } t \in \mathbb{N}
$$

mit $\varphi(t):=\frac{1}{4} \pi t+\sqrt{t}$ falsch sind (s. [1] oder [3]). Man könnte sich fragen, ob Formeln wie (1) richtig werden können, wenn die konkrete Funktion $\varphi$ durch eine andere Funktion ersetzt wird. Dabei ist klar, dass man analytische Funktionen $\varphi$ finden könnte, so dass Formel (1) sogar ohne jeden Fehler richtig ist. Von daher muss man irgendwelche Zusatzforderungen an $\varphi$ stellen. Funktionen $\varphi \in C^{2}$, die aus rationalen Funktionen, Wurzeln und Logarithmen zusammengesetzt sind, haben die Eigenschaft, dass $\varphi^{\prime}(t)=\mathcal{O}(\varphi(t) / t)$, $\varphi^{\prime \prime}(t)=\mathcal{O}\left(\varphi(t) / t^{2}\right)$ ist. Wir zeigen nun, dass auch mit jeder solchen Funktionen $\varphi(t)$ die obige Gitterpunktformel falsch ist. Genauer zeigen wir allgemeiner:

SATz. Seien $\alpha, \beta, n$ natürliche Zahlen mit $n \geq 2$. Betrachte für festes $0 \leq A<B \leq \infty$ und für laufendes $t \in \mathbb{R}_{+}$die Gitterpunktmengen

$$
G(t):=\left\{(x, y) \in \mathbb{N}_{0}^{2} ; \alpha x^{n}+\beta y^{n} \leq t, A y \leq x \leq B y\right\} .
$$

Es gibt dann keine Funktion $\varphi \in C^{2}$ mit

$$
\begin{aligned}
|G(t)| & =\varphi(t)+\mathcal{O}\left(t^{1 / 2 n}\right) & & \text { für } t \in \mathbb{N}, \\
\varphi^{\prime}(t) & =\mathcal{O}\left(\varphi(t) t^{1 / 2 n-1}\right), & \varphi^{\prime \prime}(t)=\mathcal{O}\left(\varphi(t) t^{1 / 2 n-2}\right) & \text { für } t \in \mathbb{R}_{+} .
\end{aligned}
$$

Ist $A=0$ oder $B=\infty$, so gibt es eine solche Funktion $\varphi \in C^{2}$ nicht einmal dann, wenn auf der rechten Seite von (3) bzw. (4) die Zahl $\frac{1}{2 n}$ durch die größere Zahl $\left(1-\frac{1}{n}\right) \frac{1}{n}$ ersetzt wird. 
Beweis. Mit dem Mittelwertsatz folgt aus (4), dass (3) richtig ist auch für $t \in \mathbb{R}_{+}$. Definiere nun, für $r \in \mathbb{R}_{+}$,

$$
\begin{aligned}
\psi(r) & :=\left|G\left(r^{n}\right)\right|, \\
M & :=\left\{(x, y) \in\left(\mathbb{R}_{\geq 0}\right)^{2} ; \alpha x^{n}+\beta y^{n} \leq 1, A y \leq x \leq B y\right\} .
\end{aligned}
$$

$M$ ist eine "geeignete" Menge im Sinne von [4] (vergl. [4, Beispiel 1.3]). Natürlich haben wir bei $R M:=\left\{(x R, y R) \in \mathbb{R}^{2} ;(x, y) \in M\right\}$, dass gilt

$$
R M=\left\{(x, y) \in\left(\mathbb{R}_{\geq 0}\right)^{2} ;\left(\alpha x^{n}+\beta y^{n}\right)^{1 / n} \leq R, A y \leq x \leq B y\right\} .
$$

Sei jetzt $\left(x_{0}, y_{0}\right) \in M \cap \mathbb{R}_{+}^{2}$ ein beliebiger rationaler Punkt. Setze

$$
\gamma:=\alpha x_{0}^{n}+\beta y_{0}^{n}, \quad \widetilde{\alpha}:=\alpha / \gamma, \quad \widetilde{\beta}:=\beta / \gamma .
$$

Dann hat $\widetilde{\alpha} x^{n}+\widetilde{\beta} y^{n}=1$ die rationale Lösung $\left(x_{0}, y_{0}\right) \in M$. Wähle nun $k \in \mathbb{N}$ fest, so dass $k \widetilde{\alpha} x_{0}^{n-1} \in \mathbb{Z}, k \widetilde{\beta} y_{0}^{n-1} \in \mathbb{Z}$. Für dieses $k$ ist die Menge

$$
\mathcal{R}_{k}:=\left\{(x, y) \in \mathbb{Q}_{+}^{2} ; \widetilde{\alpha} x^{n}+\widetilde{\beta} y^{n}=1, k \widetilde{\alpha} x^{n-1} \in \mathbb{Z}, k \widetilde{\beta} y^{n-1} \in \mathbb{Z}\right\}
$$

aus [4, Satz 1] nicht leer. Somit ist also nach [4, Satz 1]

$$
\left|\sum_{(x, y) \in R M \cap \mathbb{N}_{0}^{2}} e\left(k\left(\widetilde{\alpha} x^{n}+\widetilde{\beta} y^{n}\right)^{1 / n}\right)\right| \geq \lambda_{1} R^{1+1 / 2}
$$

mit einer Konstanten $\lambda_{1}>0$. Also ist für $m:=k \gamma^{-1 / n} \in \mathbb{R}_{+}$( $m$ fest, da $k$ fest) die Beziehung

$$
\sum_{(x, y) \in R M \cap \mathbb{N}_{0}^{2}} e\left(m\left(\alpha x^{n}+\beta y^{n}\right)^{1 / n}\right)=\mathcal{O}\left(R^{1+1 / 2}\right)
$$

falsch. Betrachte nun für grosses $K$ und grosses $R$ und für jedes $j \in \mathbb{N}$ mit $1 \leq j \leq m K R$ die Menge

$$
M_{j}=\left\{(x, y) \in \mathbb{N}_{0}^{2} ; \frac{j-1}{m K}<\left(\alpha x^{n}+\beta y^{n}\right)^{1 / n} \leq \frac{j}{m K}, A y \leq x \leq B y\right\} .
$$

Es ist, mit der Funktion $\psi$ aus (5),

$$
\left|M_{j}\right|=\psi\left(\frac{j}{m K}\right)-\psi\left(\frac{j-1}{m K}\right) .
$$

Weiter folgt aus (6) und (7), dass

$$
\sum_{j=1}^{m K R} \sum_{(x, y) \in M_{j}} e\left(m\left(\alpha x^{n}+\beta y^{n}\right)^{1 / n}\right)=\mathcal{O}\left(R^{3 / 2}\right)
$$

falsch ist. Wenn für $(x, y) \in M_{j}$ die Zahl $m\left(\alpha x^{n}+\beta y^{n}\right)^{1 / n}$ durch die Zahl $j / K$ ersetzt wird, ist der Fehler $\mathcal{O}(1 / K)$. Dieser Fehler taucht $\left|M_{j}\right|$ mal auf. 
Also folgt mit (8) und (9), dass

$$
\begin{aligned}
\sum_{j=1}^{m K R} e\left(\frac{j}{K}\right)(\psi & \left.\left(\frac{j}{m K}\right)-\psi\left(\frac{j-1}{m K}\right)\right) \\
& =\mathcal{O}\left(\frac{1}{K}\right) \sum_{j=1}^{m K R}\left(\psi\left(\frac{j}{m K}\right)-\psi\left(\frac{j-1}{m K}\right)\right)+\mathcal{O}\left(R^{3 / 2}\right)
\end{aligned}
$$

falsch ist. Die Summe der rechten Seite von ist aber gleich $\psi(R)-\psi(0)$. Nach (2) bzw. (5) ist $\psi(R)=\mathcal{O}\left(R^{2}\right)$; somit ist also nach (10) die Beziehung

$$
\sum_{j=1}^{m K R} e\left(\frac{j}{K}\right)\left(\psi\left(\frac{j}{m K}\right)-\psi\left(\frac{j-1}{m K}\right)\right)=\mathcal{O}\left(R^{2} K^{-1}\right)+\mathcal{O}\left(R^{3 / 2}\right)
$$

falsch. Angenommen, es gäbe eine Funktion $\varphi$ mit (3) und (4). Nun gilt folgende Beziehung:

$$
\begin{aligned}
\sum_{j=1}^{m K R} e\left(\frac{j}{K}\right)\left[\left(\psi\left(\frac{j}{m K}\right)-\psi\left(\frac{j-1}{m K}\right)\right)\right. & \left.-\left(\varphi\left(\left(\frac{j}{m K}\right)^{n}\right)-\varphi\left(\left(\frac{j-1}{m K}\right)^{n}\right)\right)\right] \\
= & \sum_{j=1}^{m K R}\left(e\left(\frac{j}{K}\right)-e\left(\frac{j+1}{K}\right)\right)\left[\psi\left(\frac{j}{m K}\right)-\varphi\left(\left(\frac{j}{m K}\right)^{n}\right)\right] \\
& -e\left(\frac{1}{K}\right)[\psi(0)-\varphi(0)]+e\left(\frac{m K R+1}{K}\right)\left[\psi(R)-\varphi\left(R^{n}\right)\right] \\
= & \sum_{j=1}^{m K R} \mathcal{O}\left(\frac{1}{K}\right) \mathcal{O}\left(R^{1 / 2}\right)+\mathcal{O}\left(R^{1 / 2}\right)=\mathcal{O}\left(R^{3 / 2}\right) .
\end{aligned}
$$

(Hierbei wurde $\psi(r)-\varphi\left(r^{n}\right)=\left|G\left(r^{n}\right)\right|-\varphi\left(r^{n}\right)=\mathcal{O}\left(r^{1 / 2}\right)$ nach (3) benutzt.) Somit folgt aus (11), dass die Beziehung

$$
\begin{aligned}
\sum_{j=1}^{m K R} e\left(\frac{j}{K}\right)\left(\varphi\left(\left(\frac{j}{m K}\right)^{n}\right)-\varphi\left(\left(\frac{j-1}{m K}\right)^{n}\right)\right) & \\
& =\mathcal{O}\left(R^{2} K^{-1}\right)+\mathcal{O}\left(R^{3 / 2}\right)
\end{aligned}
$$

falsch ist. Nach dem Mittelwertsatz gibt es $0 \leq \theta_{j} \leq 1$, so dass die Beziehung

$$
\begin{aligned}
\sum_{j=1}^{m K R} e\left(\frac{j}{K}\right)\left(n\left(\frac{j}{m K}\right)^{n-1} \varphi^{\prime}\left(\left(\frac{j-\theta_{j}}{m K}\right)^{n}\right)\right. & \left.\frac{1}{m K}\right) \\
& =\mathcal{O}\left(R^{2} K^{-1}\right)+\mathcal{O}\left(R^{3 / 2}\right)
\end{aligned}
$$


falsch ist. Für $K \rightarrow \infty$ bei noch festem grossen $R$ ist die linke Seite von (14) aber eine Riemannsche Summe zum Integral $\int_{0}^{R} e(m t) n t^{n-1} \varphi^{\prime}\left(t^{n}\right) d t$ und unterscheidet sich bei sehr grossem $K$ von diesem Integral um weniger als $\mathcal{O}\left(R^{3 / 2}\right)$. Damit besagt (14), dass die Beziehung

$$
\int_{0}^{R} e(m t) t^{n-1} \varphi^{\prime}\left(t^{n}\right) d t=\mathcal{O}\left(R^{3 / 2}\right)
$$

falsch ist. Die linke Seite von (15) wird partiell integriert. Somit ist die Beziehung

$$
\begin{aligned}
\frac{1}{2 \pi i m} \int_{0}^{R} e(m t)\left((n-1) t^{n-2} \varphi^{\prime}\left(t^{n}\right)+n t^{2 n-2} \varphi^{\prime \prime}\left(t^{n}\right)\right) d t & \\
& -\frac{1}{2 \pi i m} e(m R) R^{n-1} \varphi^{\prime}\left(R^{n}\right)=\mathcal{O}\left(R^{3 / 2}\right)
\end{aligned}
$$

ebenfalls falsch. Damit kann aber nicht (4) gelten, weil wegen $\varphi(t) \sim t^{2 / n}$ schon nach (4)

$$
\varphi^{\prime}\left(t^{n}\right)=\mathcal{O}\left(t^{5 / 2-n}\right), \quad \varphi^{\prime \prime}\left(t^{n}\right)=\mathcal{O}\left(t^{5 / 2-2 n}\right)
$$

gelten würde und damit die Beziehung (16) doch richtig wäre. Damit ist unser Satz im Fall $0<A<B<\infty$ gezeigt.

Für den Fall, dass $A=0$ oder $B=\infty$ ist, liegt ein rationaler Punkt $\left(x_{0}, y_{0}\right) \neq(0,0)$ mit $x_{0}=0$ oder $y_{0}=0$ in der oben definierten "geeigneten" Menge $M$. Wie oben können wir dann annehmen, dass dann wieder $\left(x_{0}, y_{0}\right) \in \mathcal{R}_{k}$ für geeignetes $k \in \mathbb{N}$ ist. Wird $m$ wie oben definiert, so ist also nach $[4$, Satz 1] analog (7)

$$
\sum_{(x, y) \in R M \cap \mathbb{N}_{0}^{2}} e\left(m\left(\alpha x^{n}+\beta y^{n}\right)^{1 / n}\right)=\mathcal{O}\left(R^{1+(1-1 / n)}\right)
$$

falsch. Die gesamte anschliessende Argumentation bleibt richtig, wenn der Exponent $1 / 2$ durch $1-1 / n$ ersetzt wird, so dass damit die Behauptung auch für den Fall $A=0$ bzw. $B=\infty$ gezeigt ist.

Unser Satz ist im Vergleich zu dem Satz von Krätzel [2, 3.17.A] in der Negativaussage allgemeiner, enthält jedoch kein Äquivalent zur Positivaussage.

\section{Literatur}

[1] G. H. Hardy, On the expression of a number as the sum of two squares, Quart. J. Math. 46 (1915), 263-283.

[2] E. Krätzel, Lattice Points, Kluwer, Dordrecht, 1988.

[3] E. Landau, Über die Gitterpunkte in einem Kreis (II), Göttinger Nachr. 1915, 161171. 
[4] K. Langmann, Unlösbarkeit der Gleichung $\alpha x^{n}+\beta y^{n}=z^{n}$ und Gleichverteilung der Reste von $\sqrt[n]{\alpha x^{n}+\beta y^{n}}$, Monatsh. Math. 143 (2004), 205-227.

Mathematisches Institut

Einsteinstrasse 62

D-48151 Münster, Germany

E-mail: ina.folker@math.uni-muenster.de

Eingegangen am 13.6.2005

(5009) 